\title{
Influence and Analysis of Road Width on Modeling of Expressway Sound Source
}

\author{
Xintan $\mathrm{Ma}^{1, \text { a) }}$, Shichang Feng ${ }^{1, \text { b) }}$ \\ ${ }^{1}$ Henan University of Science and Technology, Luoyang 471003, China. \\ a)maxintan@163.com \\ b)1103444277@qq.com
}

\begin{abstract}
At present, most of the traffic noise forecasting models in the domestic and international expressway are concentrated on a line source for noise prediction, and the influence of road width on sound source modeling is rarely considered, which is different from the actual situation. And the noise prediction by the acoustic point is also less analyzed from the viewpoint of the spectral sound pressure level. In this paper, acoustic simulation software is used to simulate the four-lane, six-lane and eight-lane three kinds of expressways. The sound pressure level and comprehensive noise values at different distances from the shoulder are calculated under single and dual noise prediction models, and they have been compared and analyze from the frequency domain aspects. The results show that with the increase of pavement width, such as four-lane, six-lane and eight-lane, the difference of noise forecast between two kinds of sound source models is gradually increased. The dual sound source model is more in line with the actual situation of road traffic noise than using single sound source model. This paper provides a reference for the noise forecasting of expressway.
\end{abstract}

Keywords: Road Width, Expressway, Noise

\section{INTRODUCTION}

In recent years, the development of China's expressway construction is rapid, and the speed of the vehicles on the highway has also increased rapidly, traffic problems become increasingly prominent, it's seriously affecting the health of residents on both sides of the road, the highway traffic noise prediction has always been the focus of our research work [1].

At present, in China, the commonly used noise prediction models are the noise forecasting model proposed by the Environmental Impact Assessment Code for Highway Construction Project (Trial (JTJ 005-96)) and the road noise forecast model proposed by the US Federal Highway Administration (FHWA) [2,3]. Both types of the noise prediction models concentrated all the vehicles on the highway in a line sound source, and predicted the equivalent sound pressure level of the sound point, but less considered of the influence of road width on sound source modeling and less studied from the sound spectrum of the sound pressure level, which made a problem on reducing a peak frequency noise.

The above-mentioned sound source models both look upon all the vehicles on the road as a line of sound source for noise prediction, the paper called this sound source model "single sound source noise prediction model." This model takes less considered of the influence of road width on the modeling in the process of establishment. With the increase of the road width of four lanes, six lanes and eight lanes, the noise forecast values may deviate from the actual noise value [4]. Therefore, this paper divides the expressway into two lines of sound source, which is closer to the distribution of the actual road noise source. This prediction model is called "double sound source noise prediction model". 


\section{FUNDAMENTAL ASSUMPTION}

In the case of normal expressway, the traffic conditions on both sides of the central isolation belt are basically the same. Therefore, in this paper, the spectral characteristics of the two lines sources are supposed to the same. In the measurement, we just only need to measure one of the spectral characteristics of the two lines sources, and then the spectral characteristics of the total line source of the whole road can be obtained. In addition, it is difficult to measure the spectral characteristics of a single line sound source directly on the two-way highways, so the method of analogous measurements can be used in practice [5], namely we need to measure the spectrum characteristics of the road which has the same number of lanes with half number of highway lanes and is roughly the same with the unidirectional carriageway of the highways in traffic conditions, traffic flow, speed and environmental conditions. The frequency response curve of the reference point which is $7.5 \mathrm{~m}$ distance from the equivalent lane can be measured according to the relevant standard. This curve can be used as preliminary data [6].

In this paper, a single sound source noise prediction model and a dual sound source noise prediction model are established by using the acoustic simulation software. The frequency spectrum sound pressure levels of four received points of four lanes six lanes and eight lanes highway are calculated, then Analyze the effect of road width on sound source modeling.

\section{BASIC FORMULA}

(1) The distance calculation formula of equivalent lane. This formula is often used to calculate the distance from the received point to the equivalent lane, then we can make sure the position of the sound source.

$$
r_{\text {eq }}=\sqrt{r_{\text {far }} \times r_{\text {near }}}
$$

In the Eq. 1, $r_{e q}$ represents the distance between the receiver and the equivalent lane; $r_{\text {far }}$ represents the distance between the receiver and the centerline of the farthest lane; $r_{\text {near }}$ represents the distance between the receiver and the centerline of the nearest lane.

(2) The mutual conversion formula of sound pressure level and sound power level. This formula is used to convert the sound pressure level at the reference point into the sound power level at the sound source.

$$
L_{w}=L_{p}-10 \lg \left(\frac{Q}{4 \pi}\right)
$$

$L_{w}$ represents the sound power level at the sound source; $L_{p}$ represents the sound pressure level at the reference point; $Q$ represents ground factor, when the sound source is near the ground, $Q$ is equal to 2 , when the sound source is far from the ground, $Q$ is equal to 1 .

(3) The geometric attenuation formula of Sound pressure level. When we know the sound pressure level of the reference point, then we can use this formula to calculate the sound pressure level of the received point.

$$
L_{p}(r)=L_{p}\left(r_{0}\right)-10 \lg \left(\frac{r}{r_{0}}\right)
$$

$L_{p}(r)$ represents the sound pressure level of the received point; $L_{p}\left(r_{0}\right)$ represents the sound pressure level of the reference point.

(4) The calculation formula of comprehensive noise. If we know the spectrum sound pressure level, then we can use it to calculate integrated noise value. 


$$
L_{A}=10 \lg \left(\sum_{i=1}^{n} 10^{0.1 L_{p i}}\right)
$$

$L_{A}$ represents the integrated noise value of the received point. $n$ represents the number of $1 / 3$ octave center frequencies; $L_{p i}$ represents the sound pressure level at the i-th 1/3 octave center frequency.

\section{SIMULATION}

In this paper, three kinds of expressways (four lanes, six lanes, eight lanes) with different number of lanes are established, and the single and double source noise prediction models are applied to each expressway, and from the two aspects of the spectrum sound pressure level and the comprehensive noise value, the four points which are $20 \mathrm{~m}$, $40 \mathrm{~m}, 80 \mathrm{~m}, 160 \mathrm{~m}$ distance from the shoulder are simulated and analyzed. The results are as follows:

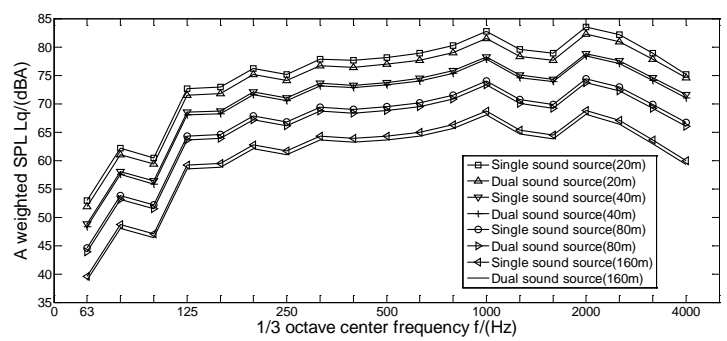

FIGURE 1. The sound pressure level spectrum diagram of the four received points in the single and dual sound source models (Four Lane)

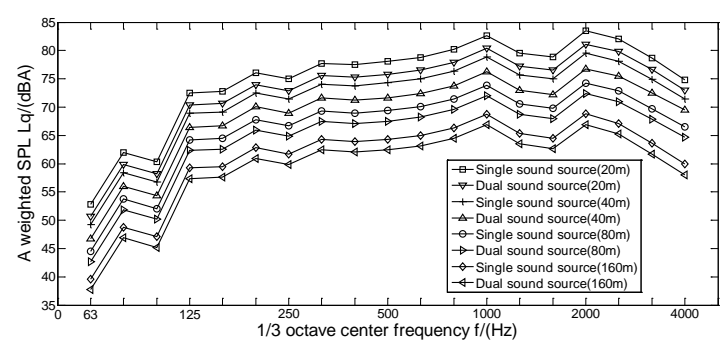

FIGURE 2. The sound pressure level spectrum diagram of the four received points in the single and dual sound source models (Six Lane)

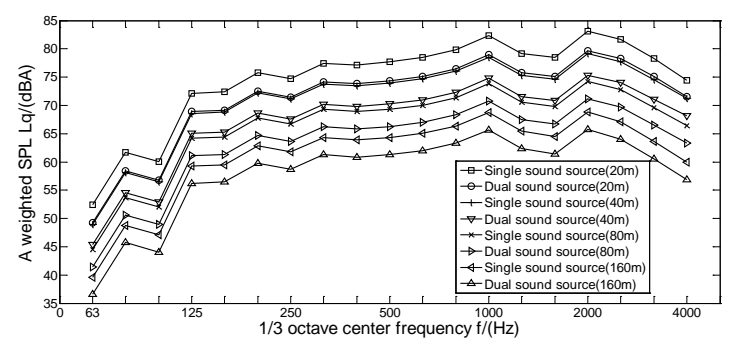

FIGURE 3. The difference of the sound pressure level of the four received points at each center frequency in the simple and dual sound source models (Eight Lane)

According to the spectrum sound pressure level and the calculation formula of comprehensive noise (Eq. 1), we can get the integrated noise value of the four received points which are $20 \mathrm{~m}, 40 \mathrm{~m}, 80 \mathrm{~m}, 160 \mathrm{~m}$ distance from the shoulder and the difference values at each center frequency in the single and dual sound source noise prediction models. As shown in Table 1. 
TABLE 1. The integrated noise value of the four received points

\begin{tabular}{|c|c|c|c|c|c|c|c|c|c|}
\hline & \multicolumn{9}{|c|}{ Integrated noise value [dB] } \\
\hline & \multicolumn{3}{|c|}{ Four-lane } & \multicolumn{3}{|c|}{ Six-lane } & \multicolumn{3}{|c|}{ Eight-lane } \\
\hline & Single & Dual & Difference & Single & Dual & Difference & Single & Dual & Difference \\
\hline $20 \mathrm{~m}$ & 91.26 & 90.07 & 1.19 & 91.17 & 88.94 & 2.23 & 90.82 & 87.47 & 3.35 \\
\hline $40 \mathrm{~m}$ & 86.81 & 86.36 & 0.45 & 87.36 & 84.76 & 2.60 & 87.01 & 83.33 & 3.68 \\
\hline $80 \mathrm{~m}$ & 82.41 & 81.74 & 0.67 & 82.34 & 80.46 & 1.88 & 82.27 & 79.19 & 3.08 \\
\hline $160 \mathrm{~m}$ & 76.99 & 76.32 & 0.67 & 77.01 & 75.14 & 1.87 & 77.04 & 73.97 & 3.07 \\
\hline
\end{tabular}

\section{CONCLUSION}

A The paper has compared and analyzed the integrated noise values and the differences of the sound pressure levels at each center frequency of the receivers which are $20 \mathrm{~m}$ or far from the shoulder, through establishing two kinds of sound source models of single and double sound sources and apply the two sound source models to three kinds of expressways. Then came to some conclusions that: The single-source model is higher than the double-source model in the noise prediction at the received point; With the increase of the road width of four lanes, six lanes and eight lanes, the noise prediction difference values of the two sound source models gradually increase. The maximum integrated noise difference value is $1.19 \mathrm{~dB}$ in the four-lane highway model, The maximum integrated noise difference value is $2.60 \mathrm{~dB}$ in the six-lane highway model, The maximum integrated noise difference value is $3.68 \mathrm{~dB}$ in the eight-lane highway model. Therefore, for the four-lane expressway, the two sound source models have almost the same effect, but for wider pavement of highway, such as six-lane and eight-lane, the dual-source model has a more accurate prediction effect than the single-source model in theory.

\section{ACKNOWLEDGMENTS}

This work was supported by the Modeling of Traffic Noise and Optimization of Acoustic Barrier under Grant No. 15A460019 and Outstanding Youth Plan for Science and Technology Innovation Talents in Henan Province under Grant No. 144100510004.

\section{REFERENCES}

1. The State Environmental Protection Administration Supervision and Management. China's Environmental Impact Assessment Training Materials [M]. Beijing: Chemical Industry Press, 2000. 269- 272.

2. Cui Bao-jun, Tong Yi, Yan Ye-wei etc. Lane Highway Traffic Noise Prediction Model Selection Research [J]. China Water Transport: Second Half, 2012,(10):68-69.

3. JTJ 005-96, Specifications for Environment Impact Assessment of Highway (on trial) [S]. 15- 16.

4. Fan Dong-Ping, Road Traffic Noise Prediction Model Analysis and Empirical Research [D]. Guangdong University of Technology, 2012: 14-15.

5. Xu Ling-lun. Highway Traffic Noise Prediction Model Application and Source of Inquiry [D]. Southwest Jiaotong University, 2011: 13-14.

6. Wang Chuan-ying, Selection Analysis of Eight Lanes of Traffic Noise Prediction Model Based on FHWA [J]. Resources Economization and Environmental Protection, 2013, (3): 79-80. 